\title{
La presencia de la Teología Católica en la Universidad de Murcia dentro del proyecto integrador de Bolonia
}

\section{The presence of Catholic theology at the University of Murcia in light of Bologna Process}

\author{
Rafael González Martín ${ }^{1}$ \\ r.gonzalezm@hotmail.com \\ Universidad de Murcia, España
}

\section{Resumen:}

En la actualidad la presencia de la teología católica en las universidades públicas españolas, - independientemente de la enseñanza de teología católica y pedagogía religiosa que se imparte en las facultades de educación dentro de los grados de maestros -, viene marcada por dos presencias muy significativa. Una de ella, viene a través de la creación de una cátedra de teología dentro del marco universitario (Cátedra de Teología en la Universidad de Granada; Cátedra de las Tres Religiones en la Universidad de Valencia; Cátedra de Teología y Ciencias de la Religión "Ignacio Ellacuría" en la Universidad Carlos III de Madrid), cuyo fin es buscar la ocasión de que la teología ofrezca su peculiar luz de conocimiento, partiendo del convencimiento de que los elementos más significativos de la tradición religiosa generan sentido y propuestas que pueden entrar en diálogo honesto con otras disciplinas.

La otra presencia es más significativa y viene establecida por la Universidad de Murcia, pues en ella, se reconoce a

Teológicos.

\begin{abstract}
:
Nowadays the presence of Catholic theology in Spanish public universities, leaving aside Catholic theology and religious education courses taught in the Faculties of Education within the education degrees, is marked by two very significant presences. One of them directly relates to the creation of chairs of theology: the Chair of Theology at the University of Granada, the Chair of the Three Religions at the University of Valencia, and the Chair of Theology and Religious Studies "Ignacio Ellacuría" at the University Carlos III in Madrid. The main purpose of these chairs is to highlight the special way with which theology looks at the production of knowledge, on the assumption that the most significant elements of religious traditions make meaning and generate proposals that may come into an honest dialogue with other disciplines. The other presence is more significant and is implemented at the University of Murcia, which recognizes theology as a discipline itself, with its own contents. Consequently, this
\end{abstract}

1 Dirección para correspondencia (correspondence address):

Rafael González Martín. Avd. Miguel de Cervantes, 26 2º-A. 41400 Écija, Sevilla (España). 
La presencia de la Teología Católica en la Universidad de Murcia dentro del proyecto integrador de Bolonia

Rafael González Martín

la teología como una disciplina propia, con materia propia y como consecuencia otorgándose por la universidad a dicho reconocimiento académico una titulación oficial. Esto es como consecuencia de la relación establecida entre el Instituto Teológico de Murcia OFM y Universidad de Murcia.

El presente trabajo tiene como objeto presentar la nueva presencia de la teología católica en el marco académico de la Universidad de Murcia como referencia del proyecto integrador de BoIonia.

\section{Palabras clave:}

Teología; Universidad pública; Centros university issues official qualifications, a fact that is made possible because of the relationship established between the OFM Theological Institute of Murcia and the University of Murcia. This work intends to explore the new presence of Catholic theology within the academic framework of the University of Murcia in reference to the Bologna Process.

Theology; State University; Theological Center.

\section{Résumé:}

Actuellement, la présence de la théologie catholique dans les universités publiques espagnoles, -indépendamment de l'enseignement de théologie catholique et de pédagogie religieuse enseignées dans les facultés d'éducation dans la formation des maîtres-, est marquée par deux présences très importantes. L'une d'elles, vient de la création d'une chaire de théologie dans le cadre universitaire (chaire de théologie-Université de Grenade, président des Trois Religions-Université de Valence, chaire de théologie et d'études religieuses «Ignacio Ellacuría»-Université Carlos III de Madrid), dont le but est de chercher à ce que la théologie ait la possibilité d'offrir sa propre lumière de connaissance, fondée sur la conviction que les éléments les plus significatifs de la tradition religieuse génèrent sens et des propositions qui peuvent entrer en dialogue honnête avec les autres disciplines.

L'autre présence est plus importante et est établie par l'Université de Murcie, car elle reconnait la théologie comme une discipline propre, avec sa propre matière et cette reconnaissance académique universitaire, par conséquent, autorise la délivrance d'un diplôme officiel. Ceci est dû à la relation établie entre I'Institut théologique OFM de Murcie et de l'Université de Murcie.

Ce travail est destiné à présenter la nouvelle présence de la théologie catholique dans le cadre académique de l'Université de Murcie comme référence de projet intégrateur de Bologne.

\section{Mots-clés:}

Théologie; Université publique; Centres théologiques.

Fecha de recepción: 20-07-2016

Fecha de aceptación: 25-01-2017 


\section{Introducción}

Es un hecho que la teología hoy, está presente en muchas universidades públicas o estatales del mundo (sobre todo en Estados Unidos y Alemania), donde prestigiosas facultades de teología ocupan un lugar natural en el concierto de los saberes universitarios.

En Europa, los institutos y facultades de teología, ya sean de universidades privadas o estatales, generalmente se benefician de subvenciones y están facultadas para otorgar diplomas reconocidos por las autoridades civiles estatales. Así, por ejemplo, tenemos las facultades de teología católica de las universidades de Lovaina y de Lovaina la Nueva, en Bélgica, las cuales están subvencionadas en su totalidad por fondos públicos. Portugal que tiene establecido en su propia Constitución el principio de separación del Estado y la Iglesia. Sin embargo, la facultad de teología católica de la universidad de Lisboa disfruta de un estatus especial desde 1990, puesto que además de estar integrada en una universidad pública, está financiada por el Estado.

En el Reino Unido, las facultades de teología, aunque independientes, están reconocidas por la universidad que otorga los diplomas o integradas en la misma. En otros países de Europa, como los países nórdicos y germánicos, los Países Bajos y Grecia, las facultades de teología forman parte integrante de las universidades estatales.

La única excepción es Francia: allí, el estudio de la teología es externo a la universidad estatal desde 1885. Sin embargo, el hecho de que en Francia no se estudie teología en las universidades estatales no deja de plantear un problema, ya que la armonización inherente al proceso de Bolonia, permite la convalidación de los diplomas canónicos en oficiales.

En España la relación entre la teología y el mundo universitario estatal quedó interrumpida, en virtud del conocido "Decreto de Libertad de Enseñanza", de 21 de octubre de 1868, en el que las facultades de teología fueron abolidas y, en consecuencia, excluidas de la enseñanza universitaria pública en España. Desde entonces el diálogo y el trabajo interdisciplinar entre la teología y los demás saberes universitarios han sido prácticamente inexistentes en el seno de las universidades públicas españolas. Mención aparte de la enseñanza de teología católica y pedagogía religiosa que se ha impartido en las escuelas de magisterio y, hoy en día, en las facultades de educación dentro de los grados de maestro. 
Esto no quiere decir que el hecho religioso y los saberes asociados a él, hayan estado ausentes de las universidades públicas. El fenómeno religioso, siempre ha estado y sigue estando presente en el tejido social de España; por lo que, ha sido objeto de estudio en la enseñanza universitaria desde no pocos puntos de vista: la cultura, la historia, la política, la sociología, el arte, la psicología y tantos otros saberes que quedan inevitablemente incompletos si de ellos arrancamos la dimensión religiosa que siempre, de una forma o de otra, ha estado y está presente en la experiencia humana y en la convivencia social. Lógicamente desde este punto de vista, al parecer, la teología forma parte de la universidad pública, como disciplina que analiza nuestra historia cultural, los fenómenos religiosos y como parte de la psicología, la sociología o la antropología. (Rodríguez Duplá 200).

En la actualidad, las universidades privadas (fundamentalmente universidades católicas) otorgan diplomas reconocidos por el Estado, a la vez que, las universidades públicas tienen autorización para crear facultades de teología católica.

\section{Marco Teórico}

El principio de libertad religiosa y de que ninguna confesión tendrá carácter estatal viene marcado por el artículo 16 de la Constitución Española de 1978 que conduce a la aconfesionalidad del Estado. Aunque los poderes públicos según recoge dicho artículo tendrán en cuenta las creencias religiosas de la sociedad española y mantendrán las consiguientes relaciones de cooperación con la Iglesia Católica y las demás confesiones. Lo que quiere decir que el Estado no toma partido ante las diferentes formas religiosas, ni se pronuncia sobre su veracidad, sino que asume la responsabilidad de ser garante y promotor de esa libertad. (Rodríguez Duplá, 2000).

Desde este supuesto, comienzan a vislumbrarse las razones por las que también un sistema político aconfesional como el nuestro debería prestar atención a las dimensiones religiosas de nuestra cultura. No se puede cercenar impunemente lo que ha crecido junto. (Rodríguez Duplá, 2000). Apartar a la teología del ámbito de saberes reconocidos y financiados por el Estado equivale a amputar una parte importante de nuestro legado cultural, y por tanto una parte importante de lo que so- 
mos. Debería cundir la idea de que al favorecer el estudio de la teología, occidente indaga en su propia racionalidad.

Podemos comprobar que la Constitución Española, pone en valor una consideración positiva del hecho religioso. Reconoce la religión como un hecho social específico y garantiza el ejercicio efectivo de la libertad religiosa por parte de los ciudadanos en privado y público. Igualmente promueve relaciones de cooperación con las instituciones religiosas (Contreras Mazario, 2008).

Luego en función de este marco legalmente constituyente se puede afirmar que la teología tiene un lugar en la UM, en función de los objetivos y fines que deben regir la universidad pública.

Objetivos y Fines de la Universidad Murcia.

La UM ofrece la siguiente declaración de intenciones, en sus estatutos, en su artículo titulado "Fundamento y objeto de la actividad universitaria":

La UM fundamenta su actividad en el principio de libertad académica, que se manifiesta en las libertades de cátedra, de investigación y de estudio, y se compromete a la consecución de los siguientes fines:

a. La creación, desarrollo, transmisión y crítica del saber mediante una docencia e investigación de calidad y excelencia.

b. La formación para el ejercicio de actividades profesionales que exijan la aplicación de conocimientos, técnicas y métodos científicos o para la creación artística.

c. La contribución al progreso y al bienestar de la sociedad mediante la producción, transferencia y aplicación práctica del conocimiento y la proyección social de su actividad.

d. La transmisión de los valores superiores de nuestra convivencia, la igualdad entre mujeres y hombres, el apoyo permanente a las personas con necesidades especiales, el fomento del diálogo, de la paz, del respeto a la diversidad cultural y de la cooperación entre los pueblos.

e. La realización de actividades de extensión universitaria dirigidas a la creación del pensamiento crítico y a la difusión de la ciencia, de la técnica y de la cultura.

f. La proyección nacional e internacional de su actividad, a través del establecimiento de relaciones con otras universidades e instituciones.

g. La promoción y conservación de su patrimonio histórico y de su 
entorno cultural, urbanístico y ambiental, como expresión de su vínculo con la sociedad."

En este sentido, deberíamos analizar la inclusión de los estudios teológicos en el repertorio de la universidad, y considerar si está perfectamente justificada su inclusión y cumple los objetivos de la institución. Entre ellos se hallan, aquellos estudios que tienen como objetivo difundir aspectos culturales esenciales de nuestra tradición occidental ligados al debate teológico, o aspectos históricos de la disciplina que tengan que ver con su origen conceptual, ya en la filosofía griega clásica, y como en sus desarrollos posteriores, o el estudio comparado de unas religiones con otras, o el análisis meta-teórico de la religión como doctrina, o el análisis de la experiencia religiosa, y posiblemente otros.

\section{Resultado}

\section{La Teología Católica en la Universidad de Murcia (UM)}

Teniendo en cuenta lo anteriormente expuesto, se analiza a continuación, la presencia de la teología dentro del marco de la UM con su programa de Máster Oficial Universitario en Teología (MUT) y el Doctorado en Teología (DT).

Los estudios de postgrado en teología tienen la intención de insertar en el ámbito universitario civil los estudios teológicos teniendo en consideración las distintas aportaciones e interrelaciones de los dos espacios del saber hasta ahora demasiado alejados. El Máster pretende una formación integral de los futuros postgraduados y atiende tanto a la formación investigadora y a la docente como a otra más importante aún en estos tiempos: la formación integral del postgraduado, a nivel humano, científico y ético.

EI MUT, que se propone, posee una relevancia propia dentro del campo teológico, especialmente en el ámbito de la especialización en el estudio y la investigación teológica, debido a la programación pensada para ofrecer a los estudiantes una formación específica en el estudio de la teología. Supone la profundización en la formación ya recibida en el Grado.

De esta manera, se cree que el MUT puede dar un sentido concreto a 
los estudios de teología en el mundo de hoy. Se trata de fijar las posiciones que la teología tiene y debe aportar al diálogo multidisciplinar en un mundo en constante evolución.

Por ello se hace necesario un MUT que permita a los estudios de teología ampliar su horizonte con el fin de ser más fieles a su misión, que no es otra que el servicio al Magisterio eclesial con el fin de «dar siempre razón de nuestra esperanza» (1 Pe 3, 15), para que el mundo crea y se salve por la fe en Jesucristo, redentor del hombre. Siguiendo las orientaciones del Concilio Vaticano II en su Decreto Optatam Totius 16: "Las disciplinas teológicas han de enseñarse a la luz de la fe y bajo la guía del magisterio de la Iglesia, de modo que los alumnos deduzcan cuidadosamente la doctrina católica de la Divina Revelación, penetren en ella profundamente, la conviertan en alimento de la propia vida espiritual, y puedan en su vida anunciarla, exponerla y defenderla. Fórmense con diligencia especial los alumnos en el estudio de la Sagrada Escritura, que debe ser como el alma de toda la teología; una vez antepuesta una introducción conveniente, iníciense con cuidado en el método de la exégesis, estudien los temas más importantes de la Divina Revelación, y en la lectura diaria y en la meditación de las Sagradas Escrituras reciban su estímulo y su alimento". (Memorias del MUT)

Además se tiene presente las orientaciones constantes del Magisterio para que la teología tenga una vertiente ecuménica clara y una perspectiva de diálogo con las otras religiones y con el mundo moderno (Cf., Unitatis Redintegratio 10).

Asimismo, según las memorias del MUT, se ha tenido presente lo que el artículo 40 de Sapientia Christiana propone como adecuado para la formación teológica, de modo que se observe un método progresivo de aprendizaje de la teología para alcanzar la madurez científica: "a) se ofrezca en primer lugar una información general, mediante la exposición coordinada de todas las disciplinas, junto con la introducción al uso del método científico (propio del Grado); b) sucesivamente se aborde con mayor profundidad el estudio de un sector particular de las disciplinas y al mismo tiempo se ejercite más de lleno a los alumnos en el uso del método de investigación científica; c) finalmente, se vaya llegando progresivamente a la madurez científica, en particular mediante la elaboración de un trabajo escrito, que contribuya efectivamente al adelanto de la ciencia". Los puntos b y c son los que se aplican al MUT.

EI MUT propuesto tiende a la formación académica holística de los 
graduados en humanidades. El objetivo es el aprovechamiento de los estudios de teología como rama del saber que permite una profundización en la historia de las ideas, la hermenéutica de los textos, el diálogo en un mundo plural y la puesta en acción de las técnicas para asumir y comprender una realidad global, compleja y plural. Esta visión integradora de las ciencias se ha abierto camino desde hace unos decenios en las universidades del ámbito americano. Allí, los estudios culturales persiguen romper la atomización del saber que se estaba produciendo por el excesivo empeño utilitarista en las ciencias humanas, derivado del utilitarismo economicista que imperaba en el ambiente académico. Mediante la integración de la literatura, los estudios sociales, las distintas artes, especialmente el cine, la antropología cultural y social y la economía política, se pretende dar una perspectiva integradora que comprenda al hombre en su mundo. Hoy debemos encaminarnos también a esta concepción. Lo vemos en la unificación de los estudios de doctorado en humanidades, que integran lo que fueron distintos doctorados. En la actualidad hay veintitrés líneas de investigación para un solo título de doctor en humanidades en la UM y la integración va en aumento, por eso mismo la teología, como disciplina y como rama del saber humano, puede y debe aportar su experiencia de siglos, como ha quedado visto arriba, y su perspectiva global de la realidad humana. (Memorias del MUT)

EI MUT es el resultado de muchos años de experiencia docente e investigadora del Instituto Teológico de Murcia1 (ITM), que ha dado como resultado la formación de buenos profesionales de la enseñanza y la investigación académica, desde la incorporación del ITM a la Facultad de Teología Fundamental de la Universidad Pontificia Antonianum (UPA) de Roma. Esta asociación permite que el ITM pueda disfrutar de la presencia de una amplia variedad de estudiantes de distintas nacionalidades y ámbitos de estudio e investigación, creando un ambiente plural e internacional en las aulas y entre el cuerpo de docentes. Esta experiencia puede suponer un plus investigador y docente y aportar el caldo de cultivo de lo que la teología quiere ser hoy en el mundo académico y científico: el punto de encuentro de diversas posturas; la unidad en medio de la diversidad; la armonía de lo heterogéneo; la cohesión en la complejidad; el vínculo de todas las posturas que florecen en este mundo global y en crisis.

EI MUT por la UM, ha sido aprobado por Agencia Nacional de Eva- 
luación de la Calidad y Acreditación (ANECA), teniendo carácter pionero: no hay otro de similares características en España y tampoco en el ámbito de estudios superiores europeo (excepción hecha de Alemania). Este carácter innovador impide poder evidenciar referencias explícitas tanto nacionales como internacionales de un MUT en facultades civiles. Sí que existen los referentes de facultades de teología eclesiásticas que están realizando másteres específicos de alguna de las ramas de los estudios teológicos: Máster en Discernimiento Vocacional y Acompañamiento Espiritual de la Universidad Pontificia de Comillas, Máster en Teología Ecuménica y Diálogo Interreligioso de la Universidad Pontificia de Salamanca, y Máster en Formación de la Universidad Pontificia Antonianum de Roma.

En el ámbito propio de la teología, la realización del MUT debe servir para que cualquiera que lo curse sea capaz de apreciar el potencial de la teológica para hacerse cargo de uno de los rasgos más característicos de las sociedades multiculturales y multirreligiosas de nuestra época: el hecho de conceder un papel relevante a las "estructuras de sentido" como es el caso de la religión, hasta el punto de que las religiones han cobrado una relevancia insospechada hace unas décadas en nuestras sociedades.

La teología puede aportar sus conocimientos, tanto de sí misma como en las conexiones que establece con otros espacios del saber. En sí misma, porque los propios estudios de teología en el MUT incluyen un abanico impresionante de formación: metodología, hermenéutica, historia, arte, filosofía, ciencias naturales, ciencias sociales, literatura, fenomenología, religiones, secularización, estudios culturales...; en las conexiones con otros espacios del saber, porque hoy día la teología es el saber integrador por excelencia. Basta repasar cualquier elenco de publicaciones teológicas para constatar la pluralidad y diversidad de las mismas en su intento de establecer un diálogo fructífero con todos los campos de conocimiento. Puede verse el número ingente de publicaciones en torno a la teología y la ciencia, la teología y las distintas ramas de la antropología, la teología y la literatura, la teología y el mundo antiguo, la teología y la historia, la teología y la filosofía, la teología y el arte y la cultura actual.

Todo esto lleva a creer que la existencia del MUT (especialmente si se hace desde una universidad civil), está sobradamente justificado como modo de volver al origen de lo que fue el nacimiento de los estudios universitarios, recuperando de alguna manera el sentido y valor etimológico de la universitas. Pero también está justificado como forma de 
mirar al futuro, un futuro que exige nuevas maneras de integración de la multiplicidad en todos los ámbitos, también en el académico. De alguna manera, esto es lo que siempre ha hecho la teología: enraizarse en la tradición como medio más eficaz de poder dar frutos en el presente y abrir la esperanza en el futuro, porque su ser es un ser en el tiempo, pero un tiempo cargado de esperanza.

Esta propuesta de título oficial de máster por la UM tiene su principal referente de adecuación en la legislación vigente preceptiva en el territorio nacional. El marco regulador justificativo de la propuesta aquí presentada está, así pues, constituido básicamente por la siguiente normativa estatal:

- Real Decreto 1125/2003, de 5 de septiembre, por el que se establece el sistema europeo de créditos y el sistema de calificaciones en las titulaciones universitarias de carácter oficial y validez en todo el territorio nacional (BOE de 18/9/03).

- Real Decreto 1393/2007, de 29 de octubre, por el que se establece la ordenación de las enseñanzas universitarias oficiales (BOE de 30/10/07). Tiene particular interés para la elaboración de esta propuesta tienen los principios generales a que hace referencia su artículo 3.5.

Para la elaboración de esta propuesta de MUT se cotejó las propuestas de similares características, salvando el hecho de que éste sería el primer MUT de una universidad civil, en centros nacionales e internacionales como:

- Universidad Pontificia Comillas: Máster en Discernimiento Vocacional y Acompañamiento Espiritual.

- Universidad Pontificia de Salamanca: Máster en Teología Ecuménica y Diálogo Interreligioso.

- Pontificia Universidad Antonianum: Máster en Formación.

- Pontificia Universidad Salesianum: Máster responsabili e coordinatori di oratorio.

Para la elaboración del plan de estudios se siguieron tanto el reglamento de la UM para la aprobación de un Máster, es decir, aprobación en los departamentos de la participación de los profesores implicados, aprobación en las juntas u órganos de gobierno de los centros implicados, aprobación en la Comisión de Estudios de Máster del Consejo de Gobierno 
y, en último término, aprobación por el propio Consejo de Gobierno de la UM; como la consulta de los órganos internos de gestión del ITM que están establecidos en los Estatutos de dicho centro: Consejo de Gestión, según los artículos 14-16; Consejo de Instituto, según los artículos 2022; Consejo de Presidencia, según los artículos 23-25; Departamentos de Teología y Filosofía.

En relación a los procedimientos de consulta internos utilizados para la elaboración y diseño de este programa de postgrado se destacaron:

- La oferta para el título del Máster fue elaborada por el Consejo de Presidencia a instancias del Consejo de Instituto y del Consejo de Gestión del ITM.

- Propuestas de profesores, a través de los departamentos de teología y filosofía para la elaboración de las distintas materias y asignaturas.

- Aprobación por el Consejo de Instituto.

- Reglamento por el que se regulan los estudios de Postgrado de la UM, documentos, normativas e información procedente de esta universidad, así como consultas específicas con La Vicerrectora de Estudios y /o la Coordinadora de Postgrado.

A partir de las estancias trimestrales de la dirección del centro en Roma y su contacto con los directores de otros centros universitarios, se fue creando la conciencia de la necesidad de integrar mejor los estudios teológicos en el ámbito universitario civil, con el fin de hacer presente la teología en la sociedad y la sociedad en la misma teología, deviniendo una relación de enriquecimiento mutuo.

Asimismo, la relación establecida entre el ITM, en especial, con las facultades de educación y letras, especialmente arte, han generado la necesidad de fomentar los vínculos existentes ofertando la posibilidad de un MUT para los alumnos de la UM.

Se ha procedido a la consulta de otros centros externos para la elaboración del plan de estudios. Estos centros son la referencia inmediata del ITM para la elaboración de sus estudios:

- Pontificia Universidad Antonianum de Roma.

- Pontificia Universidad Salesianum de Roma.

- Facultad de Educación de la Universidad de Murcia.

- Facultad de Letras de la Universidad de Murcia

También se realizó un proceso de consulta en distintas reuniones con 
profesionales en ejercicio o egresados que manifestaron la utilidad que un Máster de estas características podría aportar a la sociedad. Se recabó la opinión de los alumnos del ITM, de la Asociación de Antiguos Alumnos, de profesores de la UM que imparten docencia en este centro o que tienen relación con él, de los alumnos del máster Guía y Mantenimiento de los Bienes culturales de la Iglesia que se imparte en la UM, a los miembros de SAFAMUR (Centro de Orientación y Mediación Familiar) y a la Fundación Acción Franciscana.

Máster Oficial Universitario en Teología. (Presencial y online).

Los estudios del Máster Oficial Universitario en Teología, cumpliendo con el R.D 1393/2007, de 29 de octubre de 2007, que establece la ordenación de las enseñanzas universitarias oficiales y con el Reglamento por el que se regulan los Estudios Universitarios Oficiales de Máster y Doctorado de la UM, el Plan de Estudios de Máster Oficial Universitario en Teología, se organiza según el Sistema Europeo de Transferencia de Créditos (ECTS). Constará de 60 Créditos de 25 horas de duración cada uno a impartir a lo largo de un curso académico (dos cuatrimestres).

La organización se realiza en tres bloques formativos bien diferenciados:

- Bloque formativo A. Se pretende que el alumno adquiera la formación básica en los rudimentos de la teología, sobre todo a nivel metodológico, hermenéutico y de fundamentación de los pilares esenciales de la teología.

- Bloque formativo B. Con este bloque formativo se busca que el alumno personalice la formación que le conduce a la investigación y a la docencia.

- Bloque formativo C. Este último bloque formativo está orientado a la práctica investigadora. El alumno deberá demostrar haber adquirido todas las competencias de la titulación, mediante un trabajo de investigación original y dirigida, que concluye con la elaboración de Trabajo Fin de Máster, su presentación y defensa.

\section{Programa de Doctorado de Teología}

Se trata de un programa de doctorado unitario que comprende la formación de doctores relacionada con las Bellas Artes, la Teología y las Lenguas Clásicas y Modernas. Es un doctorado, por lo tanto, muy amplio y diverso, que ofrece posibilidades muy variadas y plurales para todos los 
que quieran acercarse a la investigación en esta rama de conocimiento y para quienes persigan un enfoque interdisciplinar en el ámbito de las Artes y Humanidades.

La línea de investigación en teología pretende que los doctorandos realicen una aplicación de los conocimientos adquiridos en el ámbito de las ciencias humanas a la investigación teológica, de modo que los expresen con claridad, e integren los campos del pensamiento propios de las Artes y Humanidades a la reflexión teológica, dentro de un diálogo fecundo y recíproco. Asimismo, se busca la integración de la teología en el mundo de la investigación académica normalizada, tal como fue desde el inicio de esta rama del saber.

\section{Conclusión}

La teología católica es un patrimonio universal que no ha de ser recluido en los límites de la comunidad religiosa católica como ha venido sucediendo hasta ahora, ya que es y ha sido, una realización cultural de primera magnitud en la historia pasada y actual, con una influencia nada despreciable en millones de personas. En ella están presentes insospechadas virtualidades para entender la vida, el mundo, la historia, las relaciones en el ámbito privado y en el social.

Por ello, parece razonable que la teología forme parte del mundo académico de las universidades públicas españolas, de manera que permita un diálogo interdisciplinario, desde el rigor que debe caracterizar el saber académico universitario, con el objetivo de que vuelva a estar en el lugar que nunca debió dejar de ocupar: el ámbito de reflexión académica y civil. De aquí, el planteamiento de que la universidad pública incluya a la teología católica como parte de sus titulaciones oficiales.

Esta propuesta está posibilitada por los vigentes acuerdos de 1979 entre el Estado Español y la Santa Sede, para el caso de las voces discrepantes de que se trata de un planteamiento confesional. El planteamiento confesional hoy en día está solventado desde el citado acuerdo que permite y exige la implicación concertada de ambas partes Universidad e Iglesia. El principio de libertad religiosa y de que ninguna confesión tendrá carácter estatal viene marcado por el artículo 16 de la Constitución Española de 1978 que conduce a la aconfesionalidad del Estado, aunque los poderes públicos según recoge dicho artículo tendrán en 
La presencia de la Teología Católica en la Universidad de Murcia dentro del proyecto integrador de Bolonia

Rafael González Martín

cuenta las creencias religiosas de la sociedad española y mantendrán las consiguientes relaciones de cooperación con la Iglesia Católica y las demás confesiones. Lo que quiere decir que el Estado no toma partido ante las diferentes formas religiosas, ni se pronuncia sobre su veracidad, sino que asume la responsabilidad de ser garante y promotor de esa libertad.

En relación a su fórmula de presencia dentro del marco universitario, lógicamente la fórmula de facultad o instituto de teología, sería la más completa y ofrecería más posibilidades de estudio e investigación. Fórmulas que existen en países europeos y que desde esta perspectiva supondría la plena integración de la universidad pública española en el proyecto de Bolonia.

Este carácter innovador de la UM pone referencias explícitas a nivel nacional de la presencia de la teología en las universidades públicas españolas.

\section{Referencias}

Anuario Académico 2008-2009 del Instituto Teológico de Murcia OFM. Recuperado de http://www.antonianumroma.org/public/pua/dispense/ITFMurcia_anuario08-09.pdf

Anuario Académico 2009-2010 del Instituto Teológico de Murcia OFM. Recuperado de http://www.antonianumroma.org/public/pua/dispense/ITFMurciaANUARIO-2009-10.pdf

Anuario Académico 2010-2011 del Instituto Teológico de Murcia OFM. Recuperado de http://www.antonianumroma.org/public/pua/dispense/ITFMurciaANUARIO10-11. pdf

Anuario Académico 2011-2012 del Instituto Teológico de Murcia OFM. h Recuperado de ttp://www.antonianumroma.org/public/pua/dispense/ITFMurcia_anuario11-12.pdf

Anuario Académico 2013-2014 del Instituto Teológico de Murcia OFM. Recuperado de http://www.itmfranciscano.org/wp-content/uploads/2013/09/ANUARIO\%2020132014.pdf

Anuario Académico 2014-2015 del Instituto Teológico de Murcia OFM. Recuperado de http://www.itmfranciscano.org/wp-content/uploads/anuario14-15.pdf

Blázquez, R. (1979). La Teología en España y la Constitución «Sapientia Christiana», en SO 6-7 (1978-79), 263-276.

Cordovilla Pérez, Á. (2012). Cristianismo, Teología y Universidad: ¿De la convivencia a la contraposición? Proyección: Teología y mundo actual, 247, 287-399.

Contreras Mazarío, J. M. (2008). Marco jurídico del factor religioso en España. Do- cumentos del Observatorio del Pluralismo Registro de España. Recuperado de www. observatorioreligion.es

Frápolli Sanz, M. J. (2012) ¿De qué hablan Dios y el César? El papel de los estudios de 
La presencia de la Teología Católica en la Universidad de Murcia dentro del proyecto integrador de Bolonia

Rafael González Martín

Teología en la Universidad Pública. Proyección: Teología y mundo actual, 247, 451464.

García Hervás, D. (1996). Reconocimiento Civil deTítulos y Estudios Eclesiásticos. (Comentario al Real Decreto 3/1995, de 13 de enero*). IUS CANONICUM, XXXVI, 71, 217-229. Gesteira, M. (1984). La teología en España, en B. Lauret-F.Refoulé. Iniciación a la práctica de la teología, I (pp. 340-356). Cristiandad: Madrid

González de Cardedal, O. (1982). Situación actual de la teología española. Salmanticensis, 29, 5-41.

González de Cardedal, O. (1980). Iglesia y política en la España de hoy. Salmanticensis, 30, 23-60.

González Martín, R.; Parada Navas, J. L. (2015). La presencia de la Teología Católica en las universidades públicas españolas en los albores del siglo XXI. Miscelánea ComiIlas. Revista de Ciencias Humanas y Sociales. Vol.73. No 143, 277-287.

González Martín, R.; Parada Navas, J. L. (2015). La Teología Católica en el contexto académico de la Universidad de Murcia en el siglo XXI. Revista Verdad y Vida, 267, 405-420.

González Montes, A. (2003). Presentación de Acuerdos Académicos y Pastorales entre Diócesis y Universidades. Edice, p. 10

Laboa Gallego, J. M. (2006). La recepción del Vaticano II en la Iglesia española. En G. Tejerina Arias (Coord.). Concilio Vaticano II, acontecimiento y recepción: estudios sobre el Vaticano II a los cuarenta años de su clausura (pp. 109-126). Salamanca: Universidad Pontificia de Salamanca.

Laboa Gallego, J. M. (2001). La Asamblea Conjunta. La Transición de la Iglesia española. Siglo XX, 50, 4-34.

Laboa Gallego, J. M. (1991). Relaciones Iglesia-Estado. Historia 16, 181, 129-140.

Laboa Gallego, J. M. (1992). Los Seminarios españoles a finales del siglo XIX. Miscelánea Comillas: Revista de Ciencias Humanas y Sociales, Vol. 50, 96-97, 31-44.

Laboa Gallego, J. M. (1985). Pensamiento teológico español del s. XX. Cuenta y razón, 20, 151-157.

López Noguero, F. (2002). El análisis de contenido como método de investigación XXI, Revista de Educación, 4, 167-179.

Luna, S. (2011). Magisterio-Teología. Relación, esquemas eclesiológicos y proceso evolutivo en el periodo 1950-1975. Revista Teología • Tomo XLVII, 6, 413-429.

Martín de Santa Olalla Saludes, P. (2007). El concordato que nunca llegó a renovarse. Revista Historia del presente, 10, 2007/2 II época, 41-59.

Montero, F. (2007). La Iglesia en el tardo franquismo o el "despegue" de la Iglesia. Revista Historia del presente, 10, 2007/2 II época, 41-59.

Nicolau, M. (1967). Reforma de las Facultades eclesiásticas, Ecclesia, 27 de febrero 1967, 25-26.

Otaduy, J. (2001). Teología en la Universidad. Régimen legal de la enseñanza religiosa durante el Franquismo y la Transición. Anuario de Historia de la Iglesia, 10, 75-94.

Palomares Montero, D.; García Aracil, A.; Castro Martínez, E. (2012). Mi- siones Actuales de la Universidades Públicas: Una Perspectiva Sociológica. ARBOR Ciencia, Pensamiento y Cultura Vol. 188 - 753 enero-febrero, 171-192. 
La presencia de la Teología Católica en la Universidad de Murcia dentro del proyecto integrador de Bolonia

Rafael González Martín

Pérez Tapias, J. A. (2012). Los usos de la razón en la Universidad. Proyección: Teología y mundo actual, 247, 401-432.

Rodríguez Duplá, L. (2000). El lugar de la Teología en la Universidad. Docu- mentos del Instituto de Antropología y Ética. Boletín del Departamento de Pastoral Universitaria y Pastoral de la Cultura. Conferencia Episcopal Española, 1, 13-21.

Romera Oñate, L. (2013). La razón responsable y la Universidad. El lugar de la teología. Instituto de Antropología y Ética y el grupo de investigación "Ciencia, razón y Fe" (CRYF), en la Universidad de Navarra.

\section{Nota}

\section{Instituto Teológico de Murcia OFM}

El Instituto Teológico de Murcia (ITM), fundado por la Provincia Franciscana de Cartagena OFM, con sede central en Murcia, es un organismo de la Conferencia Franciscana de España (CONFRES). Como centro agregado a la facultad de teología de la Pontificia Universidad Antonianum, está al servicio de la Orden Franciscana y de la diócesis de Cartagena (España), y se propone el estudio de la revelación, con los métodos propios de la ciencia teológica, según las orientaciones del Concilio Vaticano II y las directrices del magisterio de la Iglesia, en diálogo con el patrimonio filosófico perennemente válido, atento a las instancias de las culturas contemporáneas y a las exigencias de la "Nueva Evangelización" (Estatutos, 1- 4).

El Centro Superior de Teología, de la Provincia Franciscana de Cartagena nace para la formación de sus alumnos clérigos, existió de forma estable desde principios del siglo XX en Orihuela (Alicante), hasta su posterior traslado a la ciudad de Murcia. Anejo a él ha funcionado un Instituto de Ciencias Teológicas y Catequéticas, titulado "Mater Ecclesiae" dirigido por la Orden Franciscana, para la formación de religiosas/os y laicos. Filial de este Instituto es la Escuela de Iniciación Teológica (EIT) de Murcia. El Instituto "Mater Ecclesiae" obtuvo la aprobación de la CONFER Femenina Nacional Española el 26 de junio de 1971, así como una Carta laudatoria de la Congregación para los Religiosos e Institutos Seculares de fecha 20 de junio de 1972, y otra de la Comisión Episcopal de Enseñanza de 20 de junio de 1973, autorizando los estudios de dicho Instituto.

El Centro Superior de Teología, solicita el 28 de septiembre de 1977 su integración en la Universidad Pontificia de Salamanca como Instituto Teológico de Murcia OFM para cursar el Ciclo Institucional, dependiente de la Facultad de Teología. Integración que es aprobada por la misma Universidad, como Centro Afiliado, el 28 de junio de 1978. La Congregación para la Educación Católica aprueba y concede "ad quadriennium" la Afiliación del Instituto Teológico a la Universidad Pontificia de Salamanca el 21 de noviembre de 1980 y se firman las Normae y Conventiones el 20 de diciembre entre el Magnífico Sr. Rector de la Universidad Pontificia de Salamanca y el M.R.P. Ministro Provincial de la Provincia Franciscana de Cartagena, que son ratificadas por el Emmo. y Rvdmo. Sr. Cardenal J. Baum, Prefecto de la mencionada Congregación. El 10 de octubre de 1982 tiene lugar la bendición y solemne inauguración del nuevo edificio del Instituto Teológico por el Emmo. y Rvdmo. Sr. D. Vicente Enrique y Tarancón, Cardenal Arzobispo de Madrid-Alcalá y Gran Canciller de la Universidad Pontificia de Salamanca. Con esto se pone fin a un largo trayecto de esfuerzo y proyección de un Centro Superior Univer- 
sitario para el estudio de la Teología y el pensamiento cristiano en Murcia. Concluido el período de Afiliación vigente, se solicita la renovación, para lo que se firman nuevas Normae y Conventiones el 18 de junio de 1986, que ratifica la Congregación para la Educación Católica el 4 de agosto, que - dando en la misma fecha el Instituto Afiliado a la Universidad Pontificia de Salamanca por un nuevo quinquenio, renovándose la Afiliación para otro quinquenio el 26-XII-96. El Instituto Teológico se ha regido por sus propios Estatutos, aprobados por la Universidad Pontificia de Salamanca en 1978.

En julio del año 2001 se traspasa la Afiliación del ITM a la Facultad de Teología de la Pontificia Universidad Antonianum de Roma.

El 21 de noviembre de 2006, la Congregación de Educación Católica erige el ITM como Centro Agregado a la Facultad de Teología de la PUA, "ad quinquennium", aprobando, en la misma fecha, los nuevos Estatutos por los que se regirá el Instituto en adelante. Con esta categoría académica el ITM inicia en el curso 2007-2008 el nuevo plan académico de estudios, compuesto de un quinquenio para el Bachiller en Teología (Licenciatura Civil en Estudios Eclesiásticos) y un Bienio de Licenciatura en Teología, especialidad Fundamental. El 10 de enero de 2012, fue renovada la Agregaduría y aprobados los nuevos Estatutos por la misma Congregación de Educación Católica.

Convenio entre la Universidad de Murcia y el Instituto Teológico de Murcia OFM

El día 4 de mayo de 1994 se firmó un Convenio entre la UM y el ITM, con la finalidad de realizar actuaciones conjuntas en el campo de la investigación, publicaciones y docencia. Convenio renovado cada cinco años, siendo el último firmado el 17 de enero de 2012. Con esta colaboración recíproca, se integra la actuación del ITM en el ámbito universitario desde la perspectiva de las ciencias eclesiásticas, constituyéndose en un área de conocimiento más entre las que acoge este distrito universitario. El pasado 4 de enero de 2010, se firmó un convenio específico entre la UM y el ITM por el cual se concede al ITM la capacidad para desarrollar el Máster Universitario en Teología. 
